\title{
Epileptic Seizure Predictability From Scalp EEG Incorporating Constrained Blind Source Separation
}

\author{
Javier Corsini*, Leor Shoker, Saeid Sanei, Senior Member, IEEE, and Gonzalo Alarcón
}

\begin{abstract}
Most of the methods for prediction of epilepsy recently reported in the literature are based on the evaluation of chaotic behavior of intracranial electroencephalographic (EEG) recordings. These recordings require intensive surgical operations to implant the electrodes within the brain which are hazardous to the patient. Here, we have developed a novel approach to quantify the dynamical changes of the brain using the scalp EEG. The scalp signals are preprocessed by means of an effective block-based blind source separation (BSS) technique to separate the underlying sources within the brain. The algorithm significantly removes the effect of eye blinking artifacts. An overlap window procedure has been incorporated in order to mitigate the inherent permutation problem of BSS and maintain the continuity of the estimated sources.

Chaotic behavior of the underlying sources has then been evaluated by measuring the largest Lyapunov exponent. For our experiments, we provided twenty sets of simultaneous intracranial and scalp EEG recordings from twenty patients. The above recordings have been compared. Similar results were obtained when the intracranial electrodes recorded the electrical activity of the epileptic focus. Our preliminary results show a great improvement when the epileptic focus is not captured by the intracranial electrodes.
\end{abstract}

Index Terms-Blind source separation, epilepsy, epileptic seizures, scalp, seizure prediction.

\section{INTRODUCTION}

$\mathbf{E}$ PILEPSY is the most common neurological disorder, second only to stroke. Nearly 60 million people worldwide are diagnosed with epilepsy whose hallmark is recurrent seizures [1]. These seizures occur at random to impair the normal function of the brain. Epilepsy can be treated in many cases and the most important treatment today is pharmacological. The patient takes anticonvulsant drugs in a daily basis trying to achieve a steady-state concentration in blood, chosen to provide the most effective seizure control. Surgical intervention is an alternative for carefully selected cases that are refractory to medical therapy. However, in almost $25 \%$ of the total number of patients diagnosed with epilepsy, seizures cannot be controlled by any available therapy. Furthermore, side effects from both pharmacological and surgical treatments have been reported. Although most seizures are not life

Manuscript received October 8, 2004; revised May 15, 2005. Asterisk indicates corresponding author.

*J. Corsini is with the Escuela Técnica Superior de Ingenieros de Telecomunicación (Universidad Politécnica de Madrid), Madrid 28040, Spain (e-mail: javiercr@alumnos.etsit.upm.es).

L. Shoker and S. Sanei are with Cardiff University, Cardiff CF24 3AA, U.K. (e-mail: shokerl@cardiff.ac.uk; saneis@cardiff.ac.uk).

G. Alarcón is with the Department of Clinical Neurophysiology King's College Hospital, London SE5 9RS, U.K. (e-mail: galarcon@aol.com).

Digital Object Identifier 10.1109/TBME.2005.862551 threatening, they are an unpredictable source of annoyance and embarrassment. They occur when a massive group of neurons in the cerebral cortex begins to discharge in a very organized way, leading to a temporary synchronized electrical activity that disrupts the normal activity of the brain. Sometimes, such disruption manifests itself in a brief impairment of consciousness, but it can also produce a more or less complex series of abnormal sensory and motor manifestations.

Since the discovery of the human electroencephalographic (EEG) signals by Hans Berger in 1923, the EEG has been the most commonly used instrument for clinical evaluation of brain activity. Most traditional analysis of epilepsy, based on the EEG, are focused on the detection and classification of epileptic seizures. Among them, the best method of analysis is still the visual inspection of the EEG by a highly skilled electroencephalographer. However, with the advent of new signal processing methodologies based on the mathematical theory of nonlinear dynamics, there has been an increased interest in the analysis of the EEG for prediction of epileptic seizures. The brain is assumed to be a dynamical system, since epileptic neuronal networks are essentially complex nonlinear structures and a nonlinear behavior of their interactions is, thus, expected. These methods have substantiated the hypothesis that quantification of the brain's dynamical changes from the EEG might enable prediction of epileptic seizures, while traditional methods of analysis have failed to recognize specific changes prior to seizure.

Iasemidis et al. [2] were the first group to apply nonlinear dynamics to clinical epilepsy. The main concept in their studies is that seizure represents a transition of the epileptic brain to a more ordered state and, therefore, the spatiotemporal dynamical properties of the epileptic brain are different for different clinical states. Further studies of the same group, based on the temporal evolution of the short-term largest Lyapunov exponent (a modification of the largest Lyapunov exponent to account for the nonstationarity of the EEG) for patients with temporal lobe epilepsy (TLE) [3], suggested that the EEG activity becomes progressively less chaotic as the seizure approaches. Therefore, the idea that seizures were abrupt transitions in and out of an abnormal state was substituted by the idea that the brain follows a dynamical transition to seizure for at least some kind of epilepsy. Since these pioneering studies, nonlinear methods derived from the theory of dynamical systems have been employed to quantify the changes in the brain dynamics before the onset of seizures, providing evidence to the hypothesis of a route to seizure. Lehnertz et al. [4] focused their studies on the decrease of complexity in neuronal networks prior to seizure. They used the information provided by changes in the neuronal complexity loss that summarizes the complex information content of the 
correlation dimension profiles in just a single number. Lerner [5] observed that changes in the correlation integral could be used to accurately track the onset of seizure for a patient with TLE. However, Osorio et al. [6] demonstrated that these changes in the correlation integral could be perfectly explained by changes in the amplitude and frequency of the EEG signals. Van Quyen et al. [7] found a decrease in the dynamical similarity during the period prior to seizure and that this behavior became more and more pronounced as the onset of seizure approached. Moser et al. [8] employed four different nonlinear quantities within the framework of the Lyapunov theory and found strongly significant preictal changes. Litt et al. [9] demonstrated that the energy of the EEG signals increases as seizure approaches. Later on, they presented an evidence of seizure predictability with a method based on the selection of different linear and nonlinear features of the EEG [10]. Iasemidis et al. [11], [12], using the spatiotemporal evolution of the short-term largest Lyapunov exponent, demonstrated that minutes or even hours before seizure, multiple regions of the cerebral cortex progressively approach a similar degree of chaoticity of their dynamical states. They called it dynamical entrainment and hypothesized that a number of critical sites have to be locked with the epileptogenic focus over a common period of time in order for a seizure to take place. Based on this hypothesis they presented an adaptive seizure prediction algorithm that prospectively analyzes continuous EEG recordings for prediction of temporal lobe epilepsy when only the occurrence of the first seizure is known [13].

Most of these studies for prediction of epilepsy are based on intracranial EEG recordings. Two main challenges face the previous methods in their application to scalp EEG data: 1) the scalp signals are more subject to environmental noise and artifacts than the intracranial EEG; 2) the meaningful signals are attenuated and mixed in their way via soft tissue and bone. Traditional nonlinear methods (TNM's), such as the Kolmogorov entropy or the Lyapunov exponents, may be affected by the above two difficulties and, therefore, they may not distinguish between slightly different chaotic regimes of the scalp EEG [14]. One approach to circumvent these difficulties is based on the definition of different nonlinear measures that yield better performance over the TNM for the scalp EEG. This is the approach followed by Hively et al. [15]. They proposed a method based on the phase-space dissimilarity measures (PSDM) for forewarning of epileptic events from scalp EEG. The approach of Iasemidis et al. of dynamical entrainment has also been shown to work well on scalp unfiltered EEG data for seizure predictability [16]-[18]. In our study we have followed a new approach; our aim is to apply the TNM to the scalp indirectly. This requires the underlying sources of the brain to be correctly separated with the only knowledge of the scalp EEG. The noise contamination will be then reduced and we expect to obtain signals similar to the intracranial recordings to which TNM can be applied.

The paper is organized as follows. In Section II, the blind source separation (BSS) algorithm is explained in conjunction with the nonlinear measures used. In Section III, the EEG recording procedure is explained and the results are compared with the intracranial EEG. Conclusions are provided in Section IV.

\section{METHODS}

\section{A. Preprocessing}

The scalp EEG is severely contaminated by low frequency artifacts of high amplitude produced by movements of the patient. We use a baseline removal procedure to alleviate the effect of these artifacts. This procedure is performed by a filter composed of a moving window centered at the sample under process. The signals obtained after the application of this window to the scalp EEG signals are subtracted from the original signals removing the low frequency artifacts. This neither affects the information contained in higher frequencies, nor does it remove the effect of artifacts such as eye blinks. In addition, most recordings present a 50-Hz frequency component contaminating several electrodes. Therefore, the signals are lowpass filtered to eliminate this frequency component and other high frequency components generally produced by muscular activity. A Butterworth filter of order 10 with a cutoff frequency of $45 \mathrm{~Hz}$ is used. Within this range of frequencies we still have the complete information about the signals.

\section{B. Blind Separation of Scalp EEG Signals}

After the preprocessing step, the scalp EEG is still contaminated by noise and artifacts such as eye blinks. Furthermore, the scalp EEG signals are attenuated combinations of the signals produced by neuronal networks within the brain. The original signals are attenuated and mixed in their way toward the scalp via soft tissue and bone. These properties of the scalp EEG have severe effects on the estimation of nonlinear quantities and, therefore, inconsistent measures are generally obtained. If signals similar to the intracranial ones, could be recovered with only the knowledge of the scalp EEG, TNM could be used to quantify the nonlinear dynamics of these signals and the patient would benefit, without the necessity of surgical intervention, of the predictive properties of the intracranial EEG. The extraction of these signals from the scalp recordings leads us to the concept of BSS. BSS aims to extract some latent source signals when only a set of mixtures of these signals is known. In the case of scalp EEG, the low sample frequency (around $200 \mathrm{~Hz}$ ) makes the propagation delays between the signals negligible and, thus, the combination can be considered instantaneous. We have developed a constrained BSS algorithm that efficiently estimates the underlying sources within the brain and rejects any eye blinking artifact [19].

Although nothing is assumed about the mixing process, BSS compensates this lack of knowledge with the assumption that the sources are statistically independent. This means that the joint probability density of the sources can be factorized into the product of their marginal densities, i.e., $p(\mathbf{s})=\prod_{i}^{M} p\left(s_{i}\right)$ where $s_{i}$ are the $M$ sources. In addition we assume that the observed data is linearly mixed and the mixing system does not vary with time, in that

$$
\mathbf{x}(t)=\mathbf{A} \mathbf{s}(t)
$$


where $\mathbf{A}$ is an $M \times N$ full rank matrix. The BSS problem can be defined as finding a separation matrix $\mathbf{W}$ so that the sources can be recovered from the mixtures, i.e.,

$$
\mathbf{y}(t)=\mathbf{W} \mathbf{x}(t)=\mathbf{W A s}(t)
$$

where $\mathbf{y}(\mathrm{t})$ is the estimated sources and $\mathbf{W}$ is a full rank matrix. There are numerous algorithms to compute the unmixing matrix such as the well known Infomax algorithm [20] and JADE algorithm [21]. Most BSS algorithms assume that the sources have non-Gaussian distributions, but the source distributions within the brain are yet unknown. Therefore, we choose an algorithm based on an extension to the second-order blind identification (SOBI) BSS algorithm because it makes fewer assumptions on the statistical properties of the data than other BSS algorithms.

The algorithm is based on the second-order statistics of the electrode data. The objective of SOBI is to jointly diagonalize multiple time lagged covariance matrices of the estimated sources. The estimated source covariance matrix is given by

$$
\mathbf{R}_{Y}(k)=\mathbf{W R}_{X}(k) \mathbf{W}^{T}
$$

where $\mathbf{R}_{X}(k)=E\{\mathbf{x}(t) \mathbf{x}(t-k)\}$ is the covariance of the electrode data. Following [22], the least squares (LS) estimate of $\mathbf{W}$ is

$$
J(\mathbf{W})=\arg \min _{\mathbf{W}} \sum_{k=1}^{T_{B}}\|E(k)\|_{F}^{2}
$$

where $\|\cdot\|_{F}^{2}$ is the squared Frobenius norm and $E(k)$ is the error to be minimized between the covariances of the source signals, $\mathbf{R}_{S}(k)$, and the estimated sources, $\mathbf{R}_{Y}(k)$. A suitable cost function is defined based upon minimizing the off-diagonal elements for each time block, that is

$$
\begin{aligned}
J(\mathbf{W}) & =\arg \min _{\mathbf{W}} \sum_{k=1}^{T_{B}} J_{M}(\mathbf{W})(k) \\
& =\arg \min _{\mathbf{W}} \sum_{k=1}^{T_{B}}\left\|\mathbf{R}_{Y}(k)-\operatorname{diag}\left(\mathbf{R}_{Y}(k)\right)\right\|_{F}^{2}
\end{aligned}
$$

where $\operatorname{diag}(\cdot)$ is an operator which zeros the off-diagonal elements of a matrix. Minimizing the cost function in (5) will not guarantee that the eye blinking artifact is removed since there is no constraint imposed on the cost function. Therefore, a secondorder constraint term is introduced as $J_{G}=F\left(E\left\{\mathbf{g}(t) \mathbf{y}(t)^{T}\right\}\right)$, where the nonlinear function $F(\cdot)$ is chosen based on the probability density function (pdf) of the data [23]. The nonlinear function is chosen in order to have

$$
F(g)=C(g) \approx \int_{-\infty}^{g} P_{g}(\xi) d \xi
$$

where $P_{g}(\xi)$ is the PDF and $C(g)$ is the cumulative density function (CDF) of the artifact. We choose a function that is as close as possible to the $\mathrm{CDF}$ of the artifact so that its derivative will approximate the PDF of the artifact source. Then a new cost function, $J_{T}(\mathbf{W})$, is defined as

$$
J_{T}(\mathbf{W})=\arg \min _{\mathbf{W}} \sum_{k=1}^{K}\left(J_{M}(\mathbf{W}, k)+\Lambda J_{G}(\mathbf{W})\right)
$$

where $\Lambda=\left\{\Lambda_{i i}\right\}(i=1, \ldots, N)$ is the weighted factor which is governed by the correlation between the EOG and EEG signals $\left(\mathbf{R}_{G Y}\right)$, defined as

$$
\Lambda \simeq \operatorname{Pdiag}\left(\mathbf{R}_{G Y}\right)
$$

where $P \in \mathbb{R}^{+}$is an adjustable constant. Therefore, the cost function to be minimized is $J_{T}(\mathbf{W})$. We use a gradient algorithm [24] to find a $\mathbf{W}$ that minimizes $J_{T}(\mathbf{W})$. The incremental update equation is

$$
\mathbf{W}(t+1)=\mathbf{W}(t)+\Delta \mathbf{W}(t)
$$

where $\Delta \mathbf{W}=-\mu\left(\partial J_{T}(\mathbf{W}) / \partial \mathbf{W}\right)$ is the gradient of the total cost function $J_{T}(\mathbf{W})$.

For a practical prediction algorithm, the nonlinear dynamics have to be quantified over long-term EEG recordigns. The BSS algorithm presented in the previous section is block-based, therefore, after separation the continuity has to be maintained for the entire recording. This problem turns out not to be easy due to the two inherent ambiguities of BSS: 1) we cannot determine the variances (energies) of the independent components, and 2) we cannot determine the order of the independent components.

The first ambiguity states that the sources can be estimated up to a scalar factor. Therefore, when we move from one block to another, the amplitude of the sources will be generally different and the signals can be inverted. This ambiguity can be solved as explained below, so its effect can be avoided. The nonlinear dynamics are quantified by the largest Lyapunov exponent $\lambda_{1}$. The calculation of $\lambda_{1}$ estimates for each block is based on ratios of distances between points within the block. Consequently, as long as $\lambda_{1}$ is estimated for the sources obtained by applying BSS to each block of EEG data individually, there is no need to adjust the energy of the sources.

The second ambiguity, however, affects severely our algorithm. The order in which the estimated sources appear as a result of the BSS algorithm changes from block to block. Therefore, we need a procedure to reorder the signals and maintain the continuity for the entire recording. The next section explains the approach followed in our algorithm for this purpose.

\section{Permutation Problem Solution}

We have followed an overlap window approach to maintain the continuity of the estimated sources, solving both 
indeterminacies simultaneously. Instead of dividing the EEG recordings into sequential and discontinuous blocks, we employ a sliding window of fixed length, $L$, with an overlap of $L-N$ samples $(N<L)$, and apply the BSS algorithm to the block of data within that window. Therefore, assuming that $\mathbf{X}(t)=\left[x_{1}(t), x_{2}(t), \ldots, x_{m}(t)\right]^{T}$ represents the entire scalp EEG recording, where $m$ is the number of sensor signals, $x_{i}(t)$, two consecutive windows of data are selected as $\mathbf{X}_{1}(t)=\mathbf{X}\left(t_{0}+t\right)$ and $\mathbf{X}_{2}(t)=\mathbf{X}\left(t_{0}+N+t\right)$ for $t=1, \ldots, L$, where $t_{0} \geq 0$. Therefore

$$
\mathbf{X}_{2}(t)=\mathbf{X}_{1}(t+N), \quad \text { for } t=1, \ldots, L-N \text {. }
$$

Once the BSS algorithm has been applied to $\mathbf{X}_{1}(t)$ and $\mathbf{X}_{2}(t)$, we obtain the corresponding windows of the estimated sources, $\hat{\mathbf{S}}_{1}(t)=\left[\hat{s}_{1}(t), \hat{s}_{2}(t), \ldots, \hat{s}_{n}(t)\right]^{T}$ and $\hat{\mathbf{S}}_{2}(t)=\left[\hat{s}_{1}^{\prime}(t), \hat{s}_{2}^{\prime}(t), \ldots, \hat{s}_{n}^{\prime}(t)\right]^{T}$, respectively, where $n$ is the number of sources. These two windows overlap within a time interval, but due to the inherent ambiguities of BSS $\hat{\mathbf{S}}_{1}(t)$ and $\hat{\mathbf{S}}_{2}(t)$ are not equal in this interval. Instead

$$
\hat{\mathbf{S}}_{2}(t)=\mathbf{P} \cdot \mathbf{D} \cdot \hat{\mathbf{S}}_{1}(t+N), \quad \text { for } t=1, \ldots, L-N
$$

where $\mathbf{P}$ is an $n \times n$ permutation matrix and $\mathbf{D}=$ $\operatorname{diag}\left\{d_{1}, d_{2}, \ldots, d_{n}\right\}$ is the scaling matrix. Therefore, $\hat{\mathbf{S}}_{2}(t)$ is just a copy of $\hat{\mathbf{S}}_{1}(t)$ in the overlap block, with the rows (sources) permuted, and each of them scaled by a real number $d_{i}$ that accounts for the inherent amplitude ambiguity of BSS.

We have to use a measure of similarity between the rows of $\hat{\mathbf{S}}_{1}(t)$ and $\hat{\mathbf{S}}_{2}(t)$ within the overlap region in order to solve the permutation problem and, therefore, maintain the continuity of the sources. There are many different measures of similarity between two signals, such as the Kullback-Leibler divergence, but the most straightforward one is the cross-correlation. The cross-correlation between two zero mean random signals $x(t)$ and $y(t)$ is defined as

$$
r_{x y}=E\{x(t) y(t)\}
$$

where $E\{\cdot\}$ denotes the expectation. This measure gives an idea of the similarity between $x(t)$ and $y(t)$, but its values are not bounded and depend on the amplitude of the signal. Therefore, it is preferable to use a normalization of $r_{x y}$ given by the crosscorrelation coefficient

$$
\rho_{x y}=\frac{r_{x y}}{\sigma(x) \sigma(y)}
$$

where $\sigma(x)$ and $\sigma(y)$ are the variances of $x(t)$ and $y(t)$, respectively, and it satisfies

$$
-1 \leq \rho_{x y} \leq 1 .
$$

Furthermore, if $\rho_{x y}=1$, then $y=a x$, with $a>0$, and $x(t)$ and $y(t)$ are perfectly correlated; and if $\rho_{x y}=-1$, then $y=-a x$ and $x(t)$ and $y(t)$ are perfectly anti-correlated. When the two signals have no information in common, $\rho_{x y}=0$ and they are said to be uncorrelated.

Theoretically, the BSS algorithm gives independent sources at the output. Since two independent signals are uncorrelated, if the cross-correlation coefficient $\rho$ is calculated between one row $\hat{s}_{i}(t)$ of the overlap block of $\hat{\mathbf{S}}_{1}(t)$ and all the rows $\hat{s}_{j}^{\prime}(t)$ of the overlap block of $\hat{\mathbf{S}}_{2}(t)$, we should obtain all the values equal to zero except one of them for which $\left|\rho_{i j_{0}}\right|=1$. Furthermore, the value of $j_{0}$ should be different for each row, $\hat{s}_{i}(t)$. In other words, if we define the matrix $\Gamma=\left\{\gamma_{i j}\right\}$, with $\gamma_{i j}$ equal to the absolute value of $\rho$ between the $i$ th row of the overlap segment of $\hat{\mathbf{S}}_{1}(t)$ and the $j$ th row of the overlap segment of $\hat{\mathbf{S}}_{2}(t)$, then $\Gamma=\mathbf{P}^{T}$ and the permutation problem can be solved.

Once the permutation problem has been solved, each of the signals $\hat{s}_{i}(t)$ corresponds to only one of the signals $\hat{s}_{j}^{\prime}(t)$, but the latter signals are scaled and possibly inverted versions of the former signals, due to the first inherent ambiguity of BSS. Our BSS algorithm sets the variances of the output sources to one and, therefore, $\hat{s}_{i}(t)$ and $\hat{s}_{j}^{\prime}(t)$ have both equal variance. Since the signals only share an overlap of $L-N$ samples, the energy of the overlap segment of these signals will generally be different and, therefore, can be used to solve the amplitude ambiguity. In particular

$$
\hat{s}_{i}(t+N)=\operatorname{sign}\left(\rho_{i j}\right) \frac{\sigma_{i}}{\sigma_{j}^{\prime}} \hat{s}_{j}^{\prime}(t), \quad \text { for } t=1, \ldots, L-N
$$

where $\rho_{i j}$ is calculated for $\hat{s}_{i}(t)$ and $\hat{s}_{j}^{\prime}(t)$ within the overlap segment, and $\sigma_{i}^{\prime}$ and $\sigma_{j}^{\prime}$ are the variances of $\hat{s}_{i}(t)$ and $\hat{s}_{j}^{\prime}(t)$, respectively, within the overlap segment. Consequently, the continuity of the signal $\hat{s}_{i}(t)$ can be maintained with the following assignment:

$$
\hat{s}_{i}(t+N)=\operatorname{sign}\left(\rho_{i j}\right) \frac{\sigma_{i}}{\sigma_{j}^{\prime}} \hat{s}_{j}^{\prime}(t), \quad \text { for } t=1, \ldots, L
$$

or alternatively

$\hat{s}_{i}(t+L)=\operatorname{sign}\left(\rho_{i j}\right) \frac{\sigma_{i}}{\sigma_{j}^{\prime}} \hat{s}_{j}^{\prime}(t+L-N), \quad$ for $t=1, \ldots, N$

that also solve the inherent energy ambiguity of BSS.

In practice, the estimated sources are not completely uncorrelated and, therefore, $\Gamma \neq \mathbf{P}^{T}$. However, for each row we expect to obtain only one of the elements $\gamma_{i j}$ close to 1 , for $j=j_{0}$, and the rest close to zero. Therefore, the algorithm can still be applied by choosing $\hat{s}_{j}^{\prime}(t)=\hat{s}_{j_{0}}^{\prime}(t)$ and applying (16) or (17).

\section{NONLINEAR DYNAMICS}

The EEG signals result from the complex processes that occur inside the brain. Epileptic neuronal networks are essentially complex nonlinear structures; therefore, a nonlinear behavior of their interactions can be expected, leading to the concept of the brain as a dynamical system. The EEG is a multidimensional nonstationary and nonlinear time series and 
then nonlinear methods from the theory of dynamical systems could be used to quantify its dynamics, since classical linear methods of signal processing fail to detect critical features of complex time series generated by nonlinear systems.

In principle, a nonlinear system can lie in a high-dimensional or infinite-dimensional phase space. Nonetheless, when the system comes into a steady state, portions of the phase space are revisited over the time and the system lies in a subset of the phase space with a finite and generally small dimension, called attractor. When this attractor has sensitive dependence to initial conditions (it is chaotic), it is called strange attractor and its geometrical complexity is reflected by its dimension, $D_{a}$. In practice, the system's equations are not available and we only have discrete measurements of a single observable, $u(t)$, representing the system. If the system comes into such a steady state, a $p$-dimensional phase space can be reconstructed by generating $p$ different scalar signals, $x_{i}(t)$, from the original observable, $u(t)$, and embedding them into a $p$-dimensional vector

$$
\mathbf{x}(t)=\left[x_{1}(t), x_{2}(t), \ldots, x_{p}(t)\right]^{T}
$$

According to Takens [25], if $p$ is chosen large enough, we shall generally obtain a good phase portrait of the attractor and, therefore, good estimates of the nonlinear quantities. In particular, Takens' theorem states that the embedding dimension, $p$, should be at least equal to $2 \times D_{a}+1$. The easiest and probably the best way to obtain the embedding vector $\mathbf{x}(t)$ from $u(t)$ is by the method of delays. According to this method, $p$ different time delays, $t_{0}=0, t_{1}=\tau, t_{2}=2 \tau, \ldots, t_{p-1}=(p-1) \tau$, are selected and the $p$ different scalar signals are obtained as $x_{i}(t)=x\left(t+t_{i}\right)$ for $i=0, \ldots, p-1$. If $\tau$ is chosen carefully, we obtain a good phase portrait of the attractor and, therefore, good estimates of the nonlinear measures.

Since the brain is a nonstationary system, it is never in a steady state in the strictly dynamical sense. However, it can be considered as a dynamical system that constantly moves from one steady state to another. Therefore, local estimates of nonlinear measures should be possible and the changes of these quantities should be representative of the dynamical changes of the brain. Previous studies have demonstrated a more ordered state of the epileptic brain during seizure than before or after it. Iasemidis et al. used the correlation dimension to estimate the dimension, $d$, of the ictal state [2]. The values obtained ranged between 2 and 3, demonstrating the existence of low-dimensional attractor. Therefore, an embedding dimension of seven should be enough to obtain a good image of this attractor and a good space portrait of the ictal state. Increasing the value of $p$ more than what is strictly necessary increases the effect of noise and, thus, higher values of $p$ are not recommended.

Traditional methods such as the Kolmogorov entropy, the correlation dimension or the Lyapunov exponents can be used to quantify the dynamical changes of the brain. Here a method based on the calculation of the largest Lyapunov exponent [26] is used. In a $p$-dimensional system there are $p$ different Lyapunov exponents, $\lambda_{i}$. They measure the exponential rate of convergence or divergence of the different directions in the phase space. If one of the exponent is positive, the system is chaotic. Thus, two close initial conditions will diverge exponentially in the direction defined by that positive exponent. Since these exponents are ordered, $\lambda_{1} \geq \lambda_{2} \geq \lambda_{3} \geq \cdots \lambda_{d}$, to study the chaotic behavior of a system it is enough to study the changes in the largest Lyapunov exponent, $\lambda_{1}$. Therefore, we focus on the changes in the value of $\lambda_{1}$ as the epileptic brain moves from one state to another.

A practical procedure for the estimation of $\lambda_{1}$ from a time series was proposed by Wolf et al. [27]. This procedure gives a global estimate of $\lambda_{1}$ for stationary data. Since the EEG data is nonstationary [28], the algorithm to estimate $\lambda_{1}$ from the EEG should be capable of automatically identifying and appropriately weighing the transients of the EEG signals. A modification of Wolf's algorithm is, therefore, necessary for a better estimate of the largest Lyapunov exponent. For this purpose, we have used a modification of Wolf's algorithm, proposed by Iasemidis et al. [3], which modifies mainly the searching procedure to account for the nonstationarity of the EEG data. They have called this estimate the short-term largest Lyapunov exponent $\left(S T L_{\max }\right)$. The changes of the brain dynamics can be studied by the time evolution of the $S T L_{\max }$ values at different electrode sites.

\section{EXPERIMENTAL RESULTS}

\section{A. Recording Procedure}

Simultaneous scalp and intracranial EEG recordings were used to compare the results. The intracranial recordings were obtained from multi-contact foramen ovale (FO) electrodes. Electrode bundles are introduced bilaterally through the FO under fluoroscopic guidance. The deepest electrodes within each bundle lie next to medial temporal structures, whereas the most superficial electrodes lie at or just below the FO [29]. As FO electrodes are introduced via anatomical holes, they provide a unique opportunity to record simultaneously from scalp and medial temporal structures without disrupting the conducting properties of the brain coverings by burr holes and wounds, which can otherwise make simultaneous scalp and intracranial recordings unrepresentative of the habitual EEG [30]. Simultaneously, scalp EEG recordings were obtained from standard silver cup electrodes applied according to the "Maudsley" electrode placement system [31], which is a modification of the extended 10-20 system. The advantage of the Maudsley system with respect to the standard 10-20 system is that it provides a more extensive coverage of the lower part of the cerebral convexity, increasing the sensitivity for the recording from basal subtemporal structures. Cable telemetry of 32 channels was used for data acquisition (Telefactor Beekeeper system). Data were digitised at $200 \mathrm{~Hz}$ and bandpass filtered between the frequency range of $0.3-70 \mathrm{~Hz}$. The system input range was $2 \mathrm{mV}$ and data were digitised with a 12-bit analog-to-digital converter.

\section{B. Application of the Algorithm to Scalp and Intracranial Recordings}

We used simultaneous intracranial and scalp recordings in order to compare the new results with the ones obtained with 


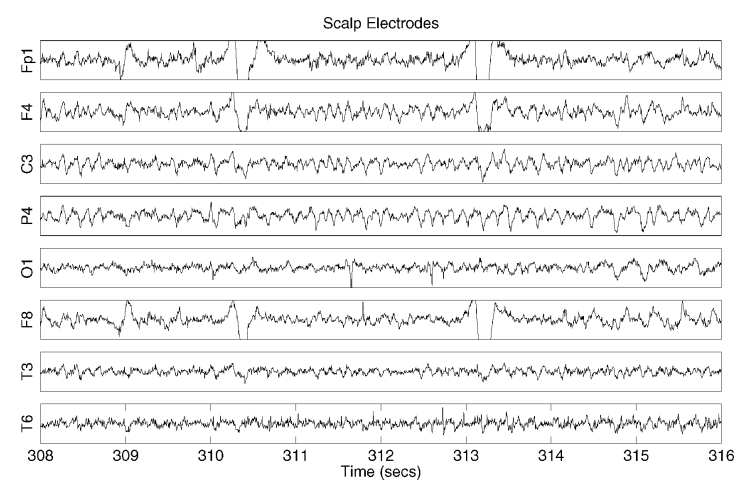

Fig. 1. EEG signal ( $8 \mathrm{~s})$ from eight out of 16 scalp electrodes during a seizure (dc removed).

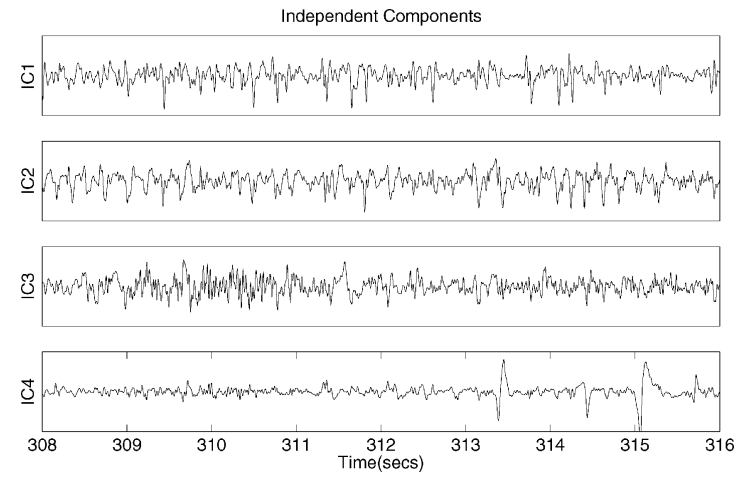

Fig. 2. Four independent components obtained by applying BBS to the scalp electrode signals shown in Fig. 1 (BBS was applied to all 16 scalp EEG's). Ictal activity is now clearly discerned in IC1 and IC2.

intracranial EEG. The scalp EEG signals were lowpass filtered and the baseline was removed. After this preprocessing, the recordings were divided into overlap windows of 2048 samples (10.24 s). For a good estimate of the correlation coefficients between the estimated sources of consecutive windows, an overlap segment of 1024 samples (5.12 s) between these windows was used. The underlying sources for each of these windows were obtained by the application of our block-based BSS algorithm. The order and amplitudes of the sources generally change from one window to another, as a result of the two inherent indeterminacies of BSS. The overlap window approach was used to maintain the continuity of the underlying sources and once the continuity was maintained, the resulting sources were divided into nonoverlapping segments of 2048 samples. The largest Lyapunov exponent, $\lambda_{1}$ was estimated for each of these segments. The intracranial signals were also divided into segments of the same size and $\lambda_{1}$ was estimated for each of these segments. In both cases, the parameters used for the estimation of $\lambda_{1}$ were those used by Iasemidis et al. for the estimation of $S T L_{\max }$, and explained in [11]. After $\lambda_{1} \mathrm{~s}$ were calculated for different segments, they were smoothed by time averaging.

Figs. 1-4 show the results obtained for a simultaneous intracranial and scalp EEG recording of $5 \mathrm{~min}$ and $38 \mathrm{~s}$ containing a focal seizure. The seizure was clearly discernible in the intracranial electrodes, around second 306, and the ictal state lasted until the end. Fig. 1 shows a segment of the signals

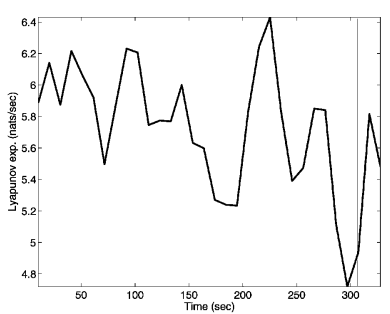

(a)

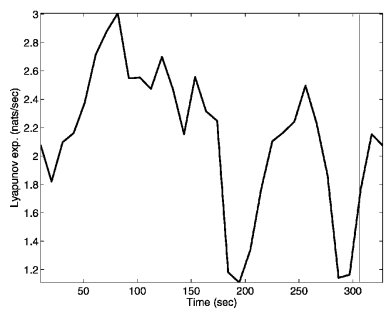

(c)

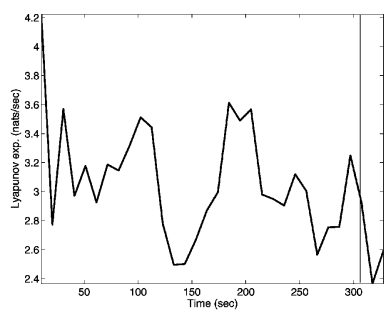

(e)

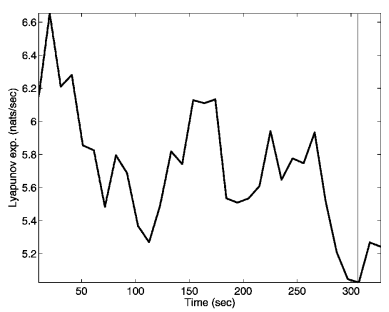

(b)

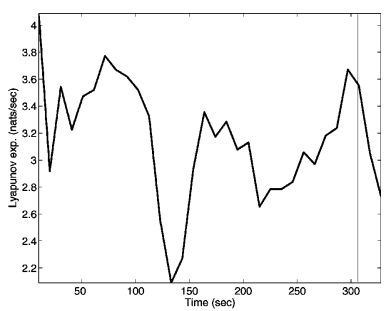

(d)

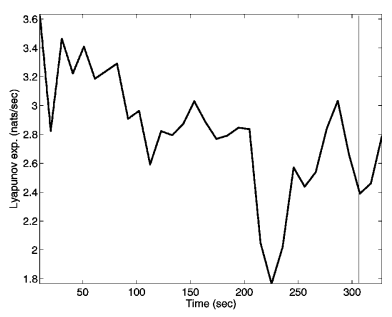

(f)
Fig. 3. The 3-point smoothed $\lambda_{1}$ evolution over time for two intracranial electrodes located in the focal area: (a) for the LF4 electrode; (b) for the LF6 electrode. (c)-(f) Smoothed $\lambda_{1}$ evolution for four scalp electrodes with the baseline removed. Length of the recording is $338 \mathrm{~s}$ and the seizure occurring at second 306 (vertical line).

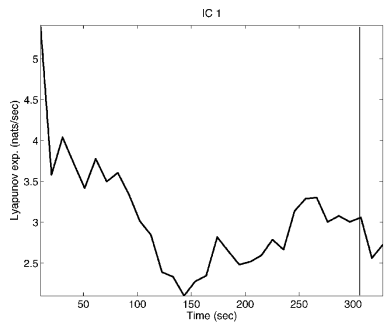

(a)

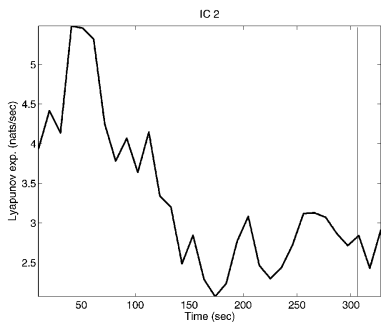

(b)
Fig. 4. The 3-point smoothed $\lambda_{1}$ evolution over time for two independent components IC1 and IC2, for which ictal activity is prominent (see Fig. 1).

recorded by the scalp electrodes during the seizure and with the baseline removed. The signals are contaminated by noise and artifacts and the seizure is not clearly discernible. Fig. 2 shows the signals obtained after applying the proposed BSS algorithm to the same segment of scalp EEG. The first and second estimated sources seem to record the seizure components while the noise and artifacts are separated in the other two sources. Fig. 3(a) and (b) illustrates the smoothed $\lambda_{1}$ variations for two intracranial electrodes located in the focal area. The smoothed $\lambda_{1}$ is calculated by averaging the current value of $\lambda_{1}$ and the previous two values. These two electrodes show a clear drop in the value of $\lambda_{1}$ at the occurrence of seizure starting prior to the onset. However, the intracranial EEG was contaminated by a 


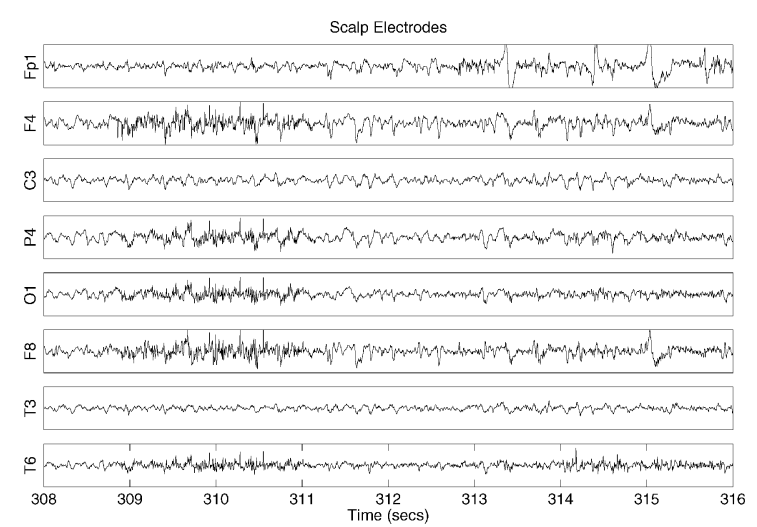

(a)
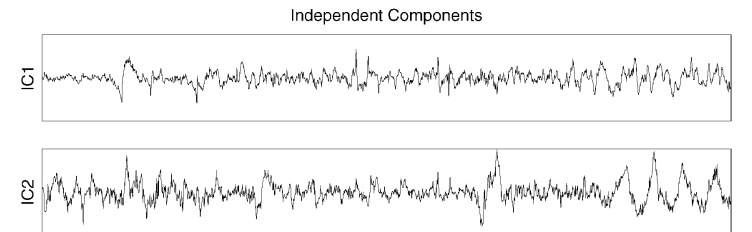

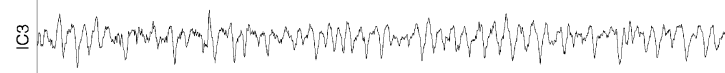

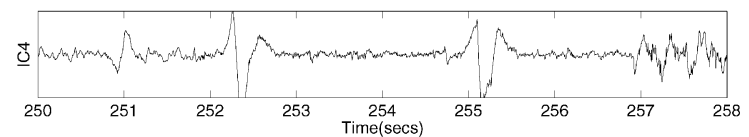

(b)

Fig. 5. (a) Segment of $8 \mathrm{~s}$ of EEG signals for eight out of 16 scalp electrodes during the seizure (dc removed). (b) The four independent components obtained by applying BBS to the scalp electrode signals shown in (a). The epileptic activity seems to be discernible in IC 3 and its $\lambda_{1}$ evolution is shown in Fig. 7.

high frequency activity that causes fluctuations of $\lambda_{1}$ for the entire recording. The scalp EEG signal was affected by many muscular artifacts due to movements of the patient. These artifacts were alleviated with the baseline removal procedure, leaving a component of high frequency muscular activity, part of which was later removed with the lowpass filter. Fig. 3(c)-(f) illustrate the smoothed $\lambda_{1}$ evolution for four scalp electrodes once the baseline was removed. The value of $\lambda_{1}$ presents large fluctuations that can be due to the presence of noise and artifacts. Although the values seem to be lower as the seizure approaches, there is not clear trend before seizure in any of the electrodes.

Fig. 4 shows the results obtained for two of the estimated sources after the application of the proposed BSS algorithm. The algorithm efficiently separates the underlying sources from the eye blinking artifacts and noise. Both figures show how the BSS algorithm efficiently separates the epileptic components. The value of $\lambda_{1}$ reaches the minimum value more than $1 \mathrm{~min}$ prior to seizure, remaining low until the end of the recording. This goes in accordance with the seizure lasting until the end of the recording.

Figs. 5-7 illustrate the results obtained from a recording of duration $5 \mathrm{~min}$ and $34 \mathrm{~s}$. In this particular case the epileptic component was not clearly visible by visual inspection of the intracranial electrode signals. The intracranial electrodes may
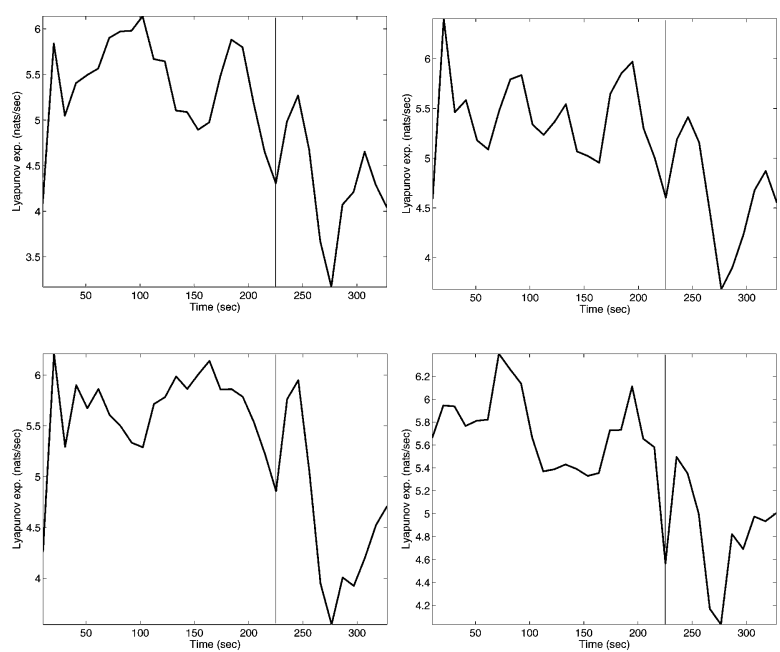

(a)

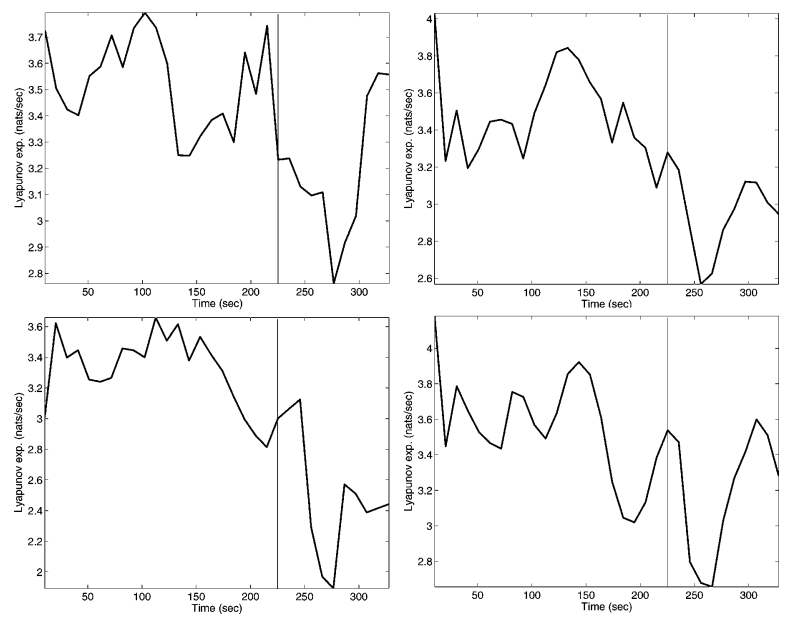

(b)

Fig. 6. (a) Intracranial EEG analysis: 3 -point smoothed $\lambda_{1}$ evolution from focal seizure \#2. In this case the electrical activity of the epileptic focus seemed not to be directly recorded by the intracranial electrodes. (b) Scalp EEG analysis: 3-point smoothed $\lambda_{1}$ evolution for four scalp electrodes with the baseline removed. The length of the recording is $334 \mathrm{~s}$, the seizure occurs at second 225 (vertical line).

not have recorded the electrical activity of the epileptic focus because of their location. Fig. 5(a) shows a segment of the signals recorded by eight scalp electrodes during that seizure. Although the signals were contaminated by noise and artifacts, the seizure components were discernible in several electrodes. Fig. 5(b) illustrates the signals obtained for the same segment of data after the BSS algorithm. In this case the seizure component seems to be separated from noise and artifacts in the third estimated source. Fig. 6(a) displays the evolution of the smoothed $\lambda_{1}$ for four different intracranial electrodes. The values fluctuate during the recording but there is a gradual drop in $\lambda_{1}$ starting at the beginning of the recording. A large drop in the value of $\lambda_{1}$ is observed for the four electrodes around second 250 and reaches to a minimum value around second 275. However, the onset of seizure occurs around second 225 and, therefore, none of the intracranial electrodes is able to predict the seizure. Fig. 6(b) shows the smoothed $\lambda_{1}$ variations for four scalp electrodes once the baseline was removed. Likewise the intracranial electrodes, 
TABLE I

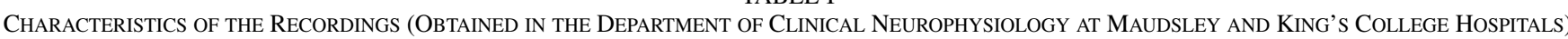

\begin{tabular}{ccccc}
\hline Type of Epilepsy & $\begin{array}{c}\text { No. of patients } \\
\text { males/females }\end{array}$ & $\begin{array}{c}\text { Age rage } \\
\text { (average) (years) }\end{array}$ & $\begin{array}{c}\text { Recording length range } \\
\text { (average) (mins.) }\end{array}$ & $\begin{array}{c}\text { No. of electrodes } \\
\text { (scalp/intracranial) }\end{array}$ \\
\hline Focal & $8 / 9$ & $11-54(27.5)$ & $5: 00-7: 20(5: 50)$ & $16 / 12$ \\
Generalized & $3 / 0$ & $33-42(36.3)$ & $4: 59-5: 55(5: 21)$ & $16 / 12$ \\
\hline
\end{tabular}

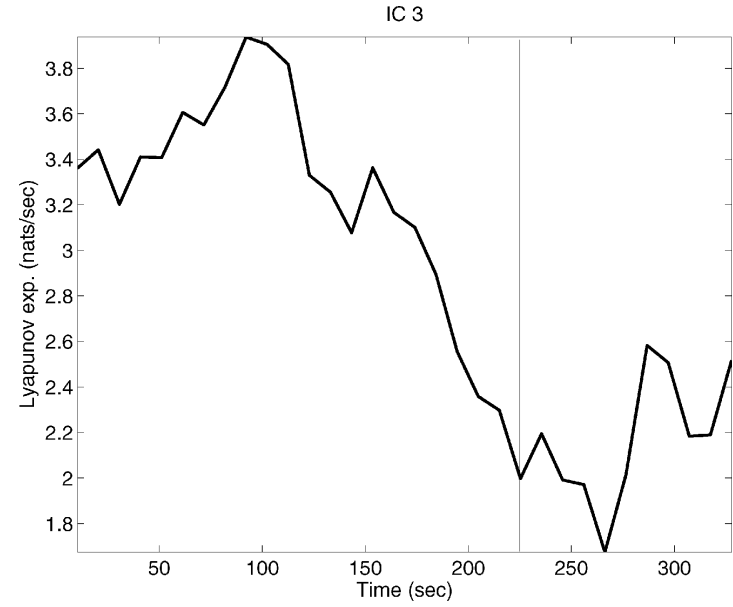

Fig. 7. The 3-point smoothed $\lambda_{1}$ evolution in a focal seizure \#2 estimated from the independent component IC3 [i.e., the one where ictal activity is discernible in Fig. 5(b)]. The electrical activity of the epileptic focus was not directly recorded by the intracranial electrodes. The length of the recording is $334 \mathrm{~s}$, the seizure occurs at second 225 (vertical line).

$\lambda_{1}$ values have large fluctuations but present a gradual drop toward seizure. Similarly, the drop to the lowest values of $\lambda_{1}$ starts after second 250 and, therefore, no prediction is possible with these electrodes.

Fig. 7 illustrates the changes in the smoothed $\lambda_{1}$ for the third estimated source obtained after the application of BSS. $\lambda_{1}$ starts decreasing approximately $2 \mathrm{~min}$ before the onset of seizure. The minimum of $\lambda_{1}$ is obtained around the same time as for the intracranial and scalp electrodes. However, a local minimum is clear at the onset of seizure and the values are clearly lower during the seizure than at the beginning of the recording. The BSS algorithm seems to separate the epileptic component in one of the estimated sources allowing the prediction of seizure even when the intracranial electrodes are not able to do it.

Fig. 8(a) and (b) shows the results obtained for a third EEG recording lasting $5 \mathrm{~min}$ and $37 \mathrm{~s}$. The electrodes recorded a generalized seizure. Fig. 8(a) illustrates the results for four intracranial electrodes. The value of $\lambda_{1}$ does not show any clear decrease until second 250 when there is a sudden drop in $\lambda_{1}$ for all the electrodes. The minimum value is obtained several seconds later; however, the onset of seizure was clearly discernible from the intracranial electrodes around second 236. Therefore, as an important conclusion, the intracranial EEG is not able to predict the onset of seizure in such cases and they are only able to detect the seizure after its onset. There is a clear drop in the value of $\lambda_{1}$ but it does not occur soon enough to predict the seizure.

Fig. 8(b) shows the results obtained after the application of BSS. The evolution of $\lambda_{1}$ is similar to the evolution for intracranial recordings. However, the drop in the value of $\lambda_{1}$ for the estimated source seems to start going down before it does for
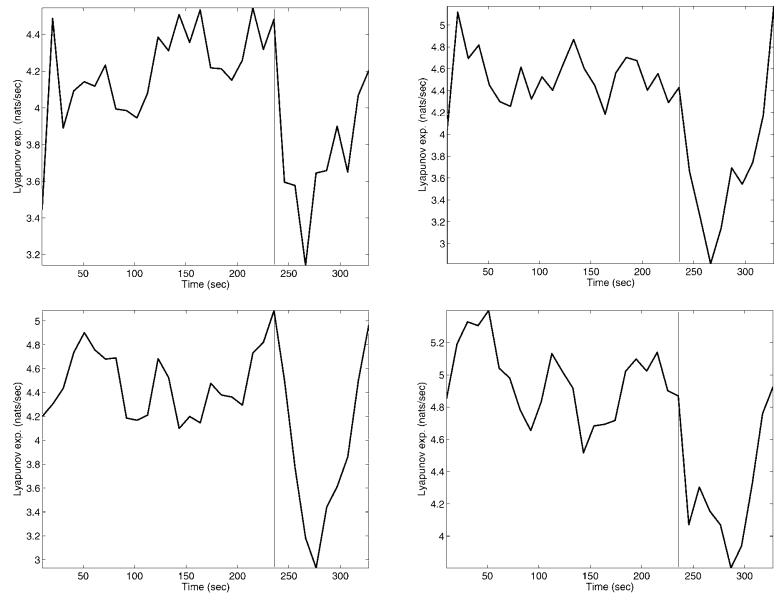

(a)

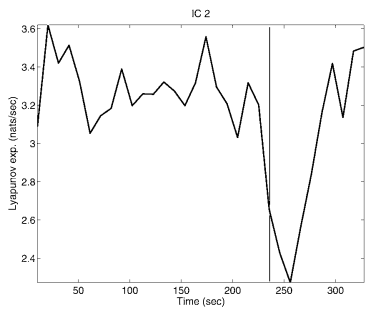

(b)

Fig. 8. (a) The 3-point smoothed $\lambda_{1}$ evolution for four intracranial electrodes in a generalized seizure. The length of the recording is $337 \mathrm{~s}$ and it records a generalized seizure starting at second 236 (vertical line). (b) The 3-point smoothed $\lambda_{1}$ evolution of IC2 component estimated from the corresponding scalp EEG. The length of the recording is $337 \mathrm{~s}$ and it records a generalized seizure at second 236 (vertical line).

the intracranial electrodes. The minimum $\lambda_{1}$ for the estimated source occurs before such a minimum for $\lambda_{1}$ is achieved for the intracranial electrodes. This means that by preprocessing the estimated sources using the proposed overall method, the occurrence time of seizure can be estimated more accurately.

For the 20 sets of the combined scalp and intracranial recordings (see Table I), 14 contained instances of focal seizure where the electrical activity of the epileptic focus was captured by the intracranial electrodes. The results obtained for the estimated sources were similar to those of intracranial recordings in 11 of these 14 cases, and the changes in the dynamics were clearly discernible before the onset of seizure for the results from both modalities. However, the estimated sources were not able to track the changes of the dynamics for one of the sources, and the continuity could not be maintained for the other two recordings due to corrupted segments within the scalp EEG. Meanwhile, intracranial EEG was able to track the changes of the dynamics for these three recordings. The intracranial electrodes did not record the electrical activity of the epileptic focus for three recordings 
and, therefore, the dynamical changes were not discernible before the onset of seizure. However, the changes of the dynamics obtained from the estimated sources were clear about $100 \mathrm{~s}$ before the onset of seizure for two of these recordings. The other three sets recorded instances of generalized seizure and none of the algorithms were able to predict the onset of seizure. The changes of the dynamics were only discernible after the onset for two of these sets.

\section{Conclusions}

The results presented in our study, although limited, appear to be promising. Scalp EEG recordings seem to contain enough information about the seizure; however, this information is mixed with the signals from the other sources within the brain and it is buried in noise and artifacts. The results of the experiments clearly show that the proposed BSS algorithm successfully separates the seizure signal (long before the seizure) from the rest of the sources, noise, and artifacts within the brain. Therefore, the well known TNM's for evaluation of the chaotic behavior of the EEG signals can be applied to them. The results obtained for the estimated source, during instances of focal epilepsy, are similar to the results obtained for intracranial electrode signals recording the electrical activity of the epileptic focus for 11 out of 14 sets of recordings. However, a clear improvement was obtained for two out of three recordings where the seizure focus was not captured by the intracranial electrodes. Scalp EEG is recorded from electrodes located over the surface of the head and, thus, record more global electrical activity of the brain. Therefore, they may capture signals emitted from many sources within the brain, although these are attenuated, mixed and hidden in noise and artifacts. BSS efficiently separates the epileptic component as one of the output sources. Prediction of seizure might be possible with these filtered scalp EEG data, even when this is not possible with intracranial EEG, especially when the epileptic activity is spread over a big portion of the brain. However, the results obtained from three sets with generalized seizure support the idea of unpredictability of this type of seizure, since they are preceded and followed by normal EEG activity. Neither preictal nor postictal states were observed for this type of seizure. Finally, although the algorithm was tested for just one seizure per patient, similar results were obtained for recordings presenting similar characteristics (type of seizure and state of the patient); therefore, the algorithm appears to be robust and we expect the results to be reproducible for seizures in the same patient.

The results obtained by this analysis, although they are very promising, are subject to several limitations. The principal limitation is due to the length of the recordings. These recordings allowed the comparison between scalp and intracranial EEG; however, they were of relatively short duration. Therefore, it was assumed that the epileptic component was active during the entire recording. Longer recordings are needed to better examine the value of BSS in the study of seizure predictability. For these longer recordings we cannot assume that the underlying sources are active during the entire recording and then the algorithm needs to detect the beginning and end of these activities. Furthermore, it was observed that the algorithm employed to maintain the continuity failed in some cases where a segment of the scalp EEG was corrupted or the electrical activity was not correctly recorded. The number of corrupted segments increases for longer recordings and, therefore, a new methodology to maintain the continuity of the estimated sources for these particular segments should be combined with the overlap window approach. Another limitation came from the fixed number of output signals selected for the BSS algorithm. We limited ourselves to a small number of estimated sources, since the BSS algorithm selects the most significant sources, and we assumed that the epileptic component was fully active for the duration of our recordings. However, for longer recordings, the number of output sources should be selected dynamically and should be equal to the number of underlying sources within the brain. The estimation of this latter number is not easy; therefore, a more practical approach is to initially select the number of output sources equal to the number of sensors. Since we expect a smaller number of sources than sensors, the BSS algorithm will separate noise components in the remaining output sources. A procedure to select the relevant sources will then be necessary. Finally, to avoid the computational complexity for long signals, an automatic procedure for detection of the onset of the prediction may be established.

\section{REFERENCES}

[1] L. D. Iasemidis, "Epileptic seizure prediction and control," IEEE Trans. Biomed. Eng., vol. 50, no. 5, pp. 549-558, May 2003.

[2] L. D. Iasemidis, H. P. Zaveri, J. C. Sackellares, W. J. Williams, and T. W. Hood, "Nonlinear dynamics of electrocorticographic data," J. Clin. Neurophysiol., vol. 5, p. 339, 1988.

[3] L. D. Iasemidis, J. C. Sackellares, H. P. Zaveri, and W. J. Willians, "Phase space topography and the Lyapunov exponent of electrocorticograms in partial seizures," Brain Topogr., vol. 2, pp. 187-201, 1990.

[4] K. Lehnertz and C. E. Elger, "Spatio-temporal dynamics of the primary epileptogenic area in temporal lobe epilepsy characterized by neuronal complexity loss," Electroencephalogr. Clin. Neurophysiol., vol. 95, pp. 108-117, 1995.

[5] D. E. Lerner, "Monitoring changing dynamics with correlation integrals: case study of an epileptic seizure," Physica D, vol. 97, pp. 563-76, 1996.

[6] I. Osorio, M. A. F. Harrison, Y. C. Lai, and M. G. Frei, "Observations on the application of the correlation dimension and correlation integral to the prediction of seizures," J. Clin. Neurophysiol., vol. 18, pp. 269-274, 2001.

[7] M. L. V. Quyen, J. Martinerie, M. Baulac, and F. J. Varela, "Anticipating epileptic seizures in real time by a nonlinear analysis of similarity between EEG recordings," NeuroReport, vol. 10, pp. 2149-2155, 1999.

[8] H. R. Moser, B. Weber, H. G. Wieser, and P. F. Meier, "Electroencephalogram in epilepsy: analysis and seizure prediction within the framework of Lyapunov theory," Physica D, vol. 130, pp. 291-305, 1999.

[9] B. Litt, R. Estellera, J. Echauz, M. D’Alessandro, R. Shor, T. Henry, P. Pennell, C. Epstein, R. Bakay, M. Dichter, and G. Vachtsevanos, "Epileptic seizures may begin hours in advance of clinical onset: a report of five patients," Neuron, vol. 30, pp. 51-64, 2001.

[10] M. D'Alessandro, R. Esteller, G. Vachtsevanos, A. Hinson, J. Echauz, and B. Litt, "Epileptic seizure prediction using hybrid feature selection over multiple intracranial eeg electrode contacts: a report of four patients," IEEE Trans. Biomed. Eng., vol. 50, no. 5, pp. 603-615, May 2003.

[11] L. D. Iasemidis, J. C. Principe, and J. C. Sackellares, "Measurement and quantification of spatio-temporal dynamics of human epileptic seizures," in Nonlinear Biomedical Signal Processing, M. Akay, Ed. Piscataway, NJ: IEEE Press, 2000.

[12] J. C. Sackellares, L. D. Iasemidis, D.-S. Shiau, R. L. Gilmore, and S. N. Roper, "Epilepsy-when chaos fails," in Chaos in the Brain?, K. Lehnertz and C. E. Elger, Eds. Singapore: World Scientific, 2000, pp. $112-133$ 
[13] L. D. Iasemidis, D. Shiau, W. Chaovalitwongse, J. C. Sackellares, P. M. Pardalos, J. C. Principe, P. R. Carney, A. Prasad, B. Veeramani, and K. Tsakalis, "Adaptive epileptic seizure prediction system," IEEE Trans. Biomed. Eng., vol. 50, no. 5, pp. 616-627, May 2003.

[14] L. M. Hively, V. A. Protopopescu, and P. C. Gailey, "Timely detection of dynamical change in scalp EEG signals," Chaos, vol. 10, pp. 864-875, 2000.

[15] L. M. Hively and V. A. Protopopescu, "Channel-consistent forewarning of epileptic events from scalp EEG," IEEE Trans. Biomed. Eng., vol. 50, no. 5, pp. 584-593, May 2003.

[16] L. D. Iasemidis, J. C. Principe, J. M. Czaplewski, R. L. Gilmore, S. N. Roper, and J. C. Sackellares, "Spatiotemporal transition to epileptic seizures: a nonlinear dynamical analysis of scalp and intracranial eeg recordings," in Spatiotemporal Models in Biological and Artificial Systems, F. Silva, J. C. Principe, and L. B. Almeida, Eds. Amsterdam, The Netherlands: IOS Press, 1997, pp. 81-88.

[17] J. C. Sackellares, L. D. Iasemidis, D. S. Shiau, R. L. Gilmore, and S. N. Roper, "Detection of the preictal transition from scalp eeg recordings," Epilepsia, vol. 40, no. S7, p. 176, 1999.

[18] D. S. Shiau, L. D. Iasemidis, W. Suharitdamrong, L. K. Dance, W. Chaovalitwongse, P. M. Pardalos, P. R. Carney, and J. C. Sackellares, "Detection of the preictal period by dynamical analysis of scalp eeg," Epilepsia, vol. 44, no. S9, pp. 233-234, 2003.

[19] L. Shoker, S. Sanei, W. Wang, and J. Chambers, "Removal of eye blinking artifact from EEG incorporating a new constrained BSS algorithm," Med. Biolog. Eng., Computing.

[20] A. J. Bell and T. J. Sejnowski, "An information-maximization approach to blind separation and blind deconvolution," Neural Computation, vol. 7, pp. 1004-1034, 1995.

[21] J.-F. Cardoso and A. Souloumiac, "Blind beamforming for non Guassian signals, "Inst. Elect. Eng. Proc.-F, vol. 140, pp. 362-370, 1993.

[22] L. Parra and C. Spence, "Convolutive blind separation of nonstationarysources," IEEE Trans. Speech Audio Process., vol. 8, no. 5, pp. 320-327, May 2000.

[23] W. Lu and J. Rajapakse, "ICA with reference," in Proc. 3rd Int. Conf. Independent Component Analysis and Blind Signal Separation (ICA2001), 2001, pp. 120-125.

[24] S. Haykin, Adaptive Filter Theory, 4th ed. Upper Saddle River, NJ: Prentice-Hall, 2002.

[25] F. Takens, "Detecting strange attractors in turbulence ," in Lectures Notes in Mathematics, Dynamical Systems and Turbulence, Warwick 1980, D. A. Rand and L.-S. Young, Eds. Berlin, Germany: SpringerVerlag, 1981, pp. 366-381.

[26] L. D. Iasemidis and J. C. Sackellares, "The temporal evolution of the largest Lyapunov exponent on the human epileptic cortex," in Measuring Chaos in the Human Brain, D. Duke and W. Pritchard, Eds. , Singapore: World Scientific, 1991, pp. 49-82.

[27] A. Wolf, J. B. Swift, H. L. Swinney, and J. A. Vastano, "Determining Lyapunov exponents from a time series," Physica D, vol. 16, pp. 285-317, 1985.

[28] N. Kawabata, "A nonstationary analysis of the electroencephalogram," IEEE Trans. Biomed. Eng., vol. BME-20, pp. 444-452, 1973.

[29] J. L. Fernández, G. Alarcón, C. D. Binnie, and C. E. Polkey, "Comparison of sphenoidal, foramen ovale and anterior temporal placements for detecting interictal epileptiform discharges in presurgical assessment for temporal lobe epilepsy," Clin. Neurophysiol. , vol. 110, pp. 895-904, 1999.

[30] D. Nayak, A. Valentín, G. Alarcón, J. J. G. Seoane, F. Brunnhuber, J. Juler, C. E. Polkey, and C. D. Binnie, "Characteristics of scalp electrical fields associated with deep medial temporal epileptiform discharges," Clin. Neurophysiol., vol. 115, pp. 1423-1435, 2004.

[31] J. H. Margerison, C. D. Binnie, and I. R. McCaul, "Electroencephalographic signs employed in the location of ruptured intracranial arterial aneurysms," Electroencephalogr. Clin. Neurophysiol., vol. 28, pp. 296-306, 1970.

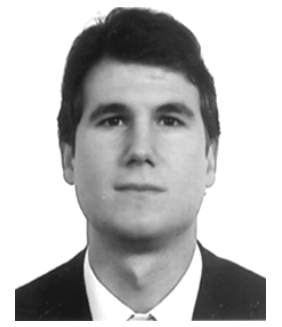

Javier Corsini was born in Madrid, Spain, in 1981. $\mathrm{He}$ received the B.S. degree in telecommunication engineering from Polytechnic University of Madrid, Spain, in 2004. He received the M.Sc. degree in Research in Digital Signal Processing (2003-2004) from King's College London, London, U.K., as an Erasmus student, focusing on epileptic seizure predictability by the application of blind source separation.

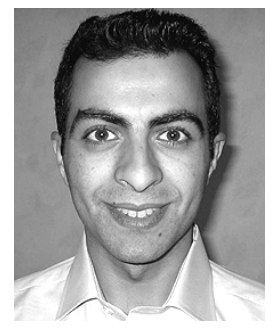

Leor Shoker is a Ph.D. degree Researcher with the Centre for Digital Signal Processing, School of Engineering, Cardiff University, Cardiff, U.K. His current research focus is brain computer interfacing (BCI) through the exploitation of blind source separation applied to EEG signals. He is also working on the development of support vector machines for classification purposes.

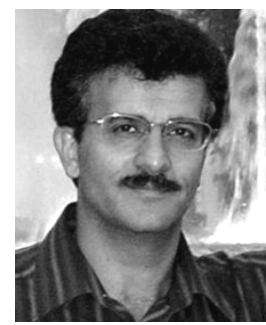

Saeid Sanei (M'97-SM'05) received the Ph.D. degree from Imperial College, London, U.K., in biomedical signal and image processing in 1991 .

He has been a member of academic staffs in Iran, Singapore, and the United Kingdom. His major interest is in biomedical signal and image processing, adaptive and nonlinear signal processing, and pattern recognition and classification. He has had a major contribution to electroencephalogram (EEG) analysis such as epilepsy prediction, cognition evaluation, and brain computer interface (BCI). He has contributed to the design and application of support vector machines (SVM) and hidden Markov models (HMM). Currently, he is a senior lecturer with the Centre for Digital Signal Processing, School of Engineering, Cardiff University.

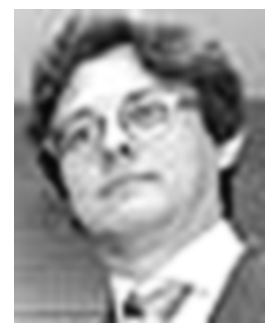

Gonzalo Alarcón received the American ECFMG Certificate for Medical Graduates from the Educational Commission for Foreign Medical Graduates (Philadelphia, PA) and the Ph.D. degree in cortical evoked potentials generated by changes in sound frequencies from the Department of Physiology, Medical School, Universidad Complutense, Madrid, Spain, in 1988.

He has contributions in EEG and ERP analysis especially for epileptic seizures. He is currently the Coordinator and Director of the M.Sc. degree course in Epileptology at Guys', King's, and St Thomas' Medical School, Department of Clinical Neurophysiology, King's College London, London, U.K. 